\title{
Operating Envelopes of Particle Sizing Instrumentation Used for Icing Research
}

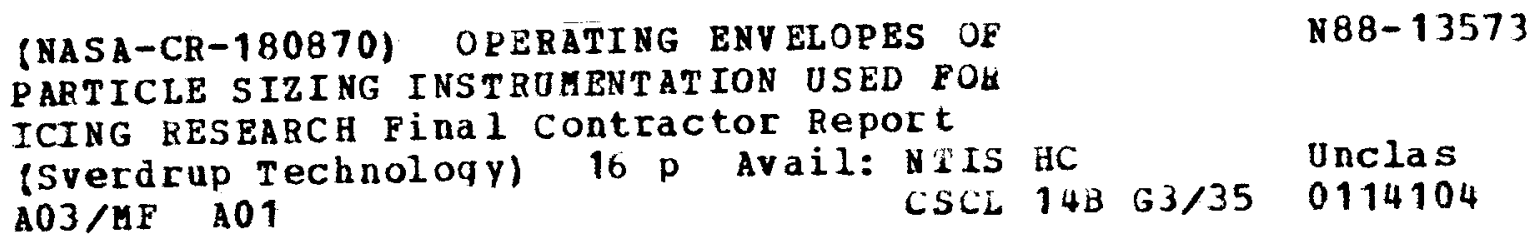

Edward A. Hovenac

Sverdrup Technology, Inc.

Lewis Research Center

Cleveland, Ohio

December 1987

Prepared for

Lewis Research Center

Under Contract NAS3-24105

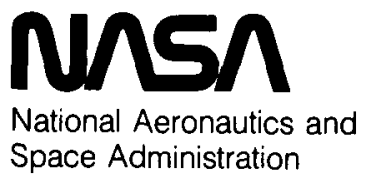

Space Administration 
OPERATING ENVELOPES OF PARTICLE SIZING INSTRUMENTATION

USED FOR ICING RESEARCH

Edward A. Hovenac

Sverdrup Technology, Inc.

Lewis Research Center

Cleveland, Ohio 44135

\section{SUMMARY}

The Forward scattering spectrometer Probe and the optical Array Probe are analyzed in terms of their ability to make accurate determinations of water droplet size distributions. Sources of counting and sizing errors are explained. The paper describes ways of identifying these errors and how these errors can affect the measurement.

\section{Introduction}

The Forward scattering spectrometer Probe (FSSP) and the Optical Array Probe (OAP) are optical particle sizing instruments used to measure the droplet size distributions (number of droplets as a function of droplet diameter), number density (number of droplets per unit volume), and liquid water content (amount of water per unit volume) in natural and artificial icing clouds. The first section of this paper is a description of basic operating principles of these instruments, followed by an explanation of how counting and sizing errors affect the various measurement quantities, as well as a definition of "operating envelope". The second and third sections give specific examples of how measurement conditions and optical effects can shape the operating envelope.

\section{Principles of operation}

Both the FSSP and the OAP are single particle counters. They count and measure the diameter of individual water droplets as they cross a laser beam. As a droplet crosses the beam several things must occur. First, the instrument must determine if the droplet is within the measurement region (also known as the probe volume). Only droplets within the probe volume can be measured accurately. Second, all droplets crossing the probe volume must be measured and grouped into size classifications called "size bins". Finally, all droplets crossing the laser beam outside of the probe volume must be rejected. The method of measuring the droplet's diameter and determining the probe volume is different for the FSSP and the OAP.

The FSSP measures the diameter of a water droplet by 
analyzing the intensity of light scattered by a droplet as it crosses the probe volume. ${ }^{1}$ The probe volume on the FSSP is a focused region of the laser beam that is about 200 micrometers in diameter and 2 millimeters in length. As a water droplet enters the probe volume it begins to scatter light. When it reaches the most intense region, in the beam center, scattering is most intense and then diminishes as the water droplet leaves the volume. The scattered light is collected by a lens system and focused onto a detector as the water droplet crosses the volume. The peak intensity on the detector (when the water droplet is in the center of the volume) is related to the size of the water droplet according to Mie scattering theory.

The OAP measures the diameter of water droplets by analyzing the image of a water droplet in the probe volume. A laser beam illuminates the water droplet as it crosses the instrument's probe volume. The image or shadow graph of the water droplet is projected through a lens system onto an array of photodiodes where the diameter of the shadow is determined. The OAP does this by counting the number of photodiodes in the shadowed region. The water droplet's diameter is related to the shadow diameter by a magnification factor.

\section{Counting and sizing Errors}

In order for a single particle counter to measure droplet size distributions correctly they must be able to measure the size of the water droplets and count them accurately. Several kinds of sizing and counting errors are examined below. These errors affect the measured size distribution differently.

Three types of sizing errors can cause shifts in the water droplet size distribution. First, the instrument may oversize or undersize all the water droplets by a constant amount. This is typically caused by shifts in the calibration. Second, the instrument may broaden the size distribution. In other words, water droplets of a single size may be grouped in size bins adjacent to the correct one. There are many reasons for this effect depending on the instrument. For example an uneven laser beam profile in the FSSP or out of focus water droplets in the OAP can reduce the precision of the instrument and cause broadening. The third sizing error is an instrument bias for any given size classification. For example in the FSSP, if the angles over which the scattered light is collected are not known precisely, then larger water droplets will have a greater error in their measured size than smaller ones.?

counting errors must also be addressed because they can cause a measurement bias as well. There are two varieties of counting errors. First, there is a bias toward counting (i.e. measuring) one size class of water droplets more than another. For example, in the OAP large water droplets are more likely to 
be seen by the instrument than smaller ones because the depth of field is greater for larger sizes. (This effect is corrected for in the instrument's software.) The second type of counting error occurs when water droplets arrive at the instrument's probe volume too rapidly to be processed. Some of the water droplets cross the probe volume unseen by the instrument because the instrument is busy analyzing the previous water droplet. This type of counting loss will not affect the size distribution or any of the calculated diameters of the water droplets (arithmetic mean, volume median etc.). This is because all the size classes of water droplets are undercounted equally. The problem occurs when number density or liquid water content is calculated. If there are counting losses, these measurements will be too low.

\section{The operating Envelope}

Any instrument must be operated within certain practical and theoretical bounds. These boundaries define the operating envelope of the instrument. If an instrument is yielding data with an acceptably small amount of error then the instrument is being operated within the envelope. The boundaries of the operating envelope are determined by measurement conditions, optical effects, and electronic limitations of the instrument. The latter of these two, optical effects and electronic limitations, are usually predictable and can be defined before making any measurements in the field. Measurement conditions are the most difficult aspect of the operating envelope to deal with. Measurement conditions can change during a test and cause the instrument to make sizing or counting errors without the operator's knowledge. The next section discusses how measurement conditions can affect an instrument's performance and ways of identifying when measurement conditions are outside the operating envelope of the instrument. This is followed by an explanation of the optical limitations of the FSSP and the OAP.

\section{Measurement Conditions}

Measurement conditions that can affect the accuracy of the FSSP and the OAP include water droplet size distribution, water droplet velocity, number density, size/velocity correlation, and the presence of ice in the cloud.

\section{Size Distribution}

When measuring a droplet distribution within a cloud or a spray there must be a match between the range of the instrument and the distribution being measured. The instrument's range should be sufficient to include the full width of the distribution. The measured distribution of sizes can indicate when the range of the instrument is matched to the size of the water droplets. It should peak toward the center of the range 
and tail off at the high and low end. Missing the ends of the distribution may cause errors in the calculated diameters. Sometimes, however, the errors are acceptable as in the case of the volume median diameter which is insensitive to errors in counting small water droplets.

\section{Velocity Errors}

Water droplets moving at high velocities through the probe volume of the FSSP can be undersized. As a water droplet passes through the probe volume, the light it scatters is focused onto a detector. The voltage output by the detector is proportional to the light scattered by the water droplet as it passes through the laser beam. For example, a water droplet moving through a probe volume with a gaussian shaped intensity profile will scatter light and the detector measuring the scattered light will put out a voltage with a gaussian profile as the water droplet crosses the probe volume. A size determination is made by measuring the peak voltage. If a water droplet passes through the beam with a high enough velocity, the electronics within the instrument cannot respond and the signal becomes degraded. 3 The signal gets spread out and the peak voltage is diminished. This results in the water droplet being undersized. This problem is not readily apparent by examining the data but can be avoided by knowing the velocity limitations of the instrument through laboratory testing. 3

For the OAP a similar sort of degradation of the signal can occur. ${ }^{4}$ In this case the problem causes counting errors. This occurs because the detectors record an image that is slightly blurred. This causes the shadow to be not as dark as when the water droplet is moving slower. The OAP interprets this as an out of focus water droplet. This effectively reduces the probe volume for fast moving water droplets. The smallest water droplets are affected most. They already have a small probe volume. If their probe volume is reduced further, it can go to zero. The small water droplets that would fall in the first size bins never get counted. This effect can be detected by observing the smallest size bins. If counts never appear in those bins, there is a strong possibility the OAP is experiencing velocity counting errors.

It should also be noted that there is a lower limit for the velocity of water droplets in the FSSP and the OAP. This limit is several meters per second. This limit precludes the use of static calibration devices.

Number Density

As with velocity, there is an upper and a lower limit for number density. Both limits can bias the data for different reasons. 
The lower limit occurs when there are not enough water droplets present in the size bins to get a statistically valid sample. As the water droplets are counted and sized they are grouped together in the size bins. As this process continues a droplet size distribution is built up. The shape of this distribution changes from one instant to the next until enough water droplets populate the size bins. Any calculations yielding the mean diameter of the water droplets cannot be made until the general shape of the distribution stabilizes. If the number density is so small or the measurement conditions are changing so rapidly that the distribution does not stabilize during the duration of the experiment, then the data will be questionable.

Extremely high number density can affect the data from the FSSP and the OAP. For both instruments, high number density causes counting losses and coincidence errors.

High number density can cause counting losses in both instruments. After a water droplet passes through the probe volume, the instrument must make a size determination. During this time (the dead time) the instrument is insensitive to water droplets crossing the probe volume and some of the water droplets are not counted. As previously mentioned this has no effect on the distribution but it does affect number density and liquid water content measurements. Corrections algorithms have been developed for the FSSP to correct these counting losses. 5

Even moderate number densities can cause counting losses if the velocity of the water droplets is high. The FSsP can detect when high number density is causing counting losses by measuring its dead time while data is being gathered. ${ }^{6}$ If the ratio of dead time to measurement time (defined as activity in the FSSP) approaches unity then counting losses are significant.

Coincidence is another error caused by high number density. It is both a sizing and a counting error that occurs when more than one water droplet is in the instrument's probe volume at a time. As number density increases the probability of coincidence errors increases. Neither the FSSP nor the OAP can detect coincidence errors. The instruments will measure two smaller water droplets as one large one. This effect tends to skew the size distribution towards larger sizes. This error is difficult to detect. One method is to measure (or estimate) the liquid water content using an independent method and use the measured distribution to calculate the number density. The reciprocal of number density gives the average volume occupied by each water droplet. This volume should be much larger than the probe volume. If it is not, then there is a high probability of coincidence errors. 


\section{Size Velocity Correlation}

A size velocity correlation occurs when water droplets in any given size bin are moving at a velocity that is different than those in the other size bins. In any single particle counter, a size velocity correlation causes data to be biased towards faster moving particles.

The reason for this bias is faster moving particles have a greater probability of crossing the probe volume than slower moving ones. ${ }^{7}$ This bias is more a property of the measurement process (called a temporal or flux sensitive measurement) than an instrument error. However, data from all single particle counters must be corrected before it can be compared with other types of instruments that are not sensitive to the water droplets' velocity. ${ }^{8}$ Also, measurements should be corrected before calculating number density or liquid water content.

For the FSSP the size velocity correlation creates one additional problem. The FSSP must have all water droplets traveling at the same velocity in order to correctly define its probe volume. One dimension of the probe volume in the FSSP is determined by the average transit time of every water droplet through the laser beam. Water droplets with transit times shorter than the average are rejected because they may have traversed the edge of the beam where the intensity is less and have the possibility of being undersized. However, the FSSP will not only reject these water droplets, but it will also reject water droplets that are moving rapidly. Both will have transit times that are shorter than the average. This effectively reduces the probe volume for fast moving water droplets. Any measurements with the FSSP in environments with a size velocity correlation should be avoided.

\section{Presence of Ice}

The FSSP performs very poorly if ice crystals are present along with the water droplets. Not only will the ice crystals be measured incorrectly, they will interfere with the measurement of the water droplets. When this occurs, the FSSP will indicate a flat size distribution indicating water droplets are populating all the size bins.9 It is theorized that the ice crystals act as tiny prisms and reflectors. Each ice crystal sends reflected and refracted rays of light in random directions. Some of these rays enter the collecting optics of the FSSP and are interpreted as water droplets. Thus one ice crystal may cause counts in many different size bins depending on its orientation in the beam.

other situations where irregularly shaped particles may be encountered is with glass beads. They can be broken, chipped or have air bubbles in them. This again could lead to counting and 
sizing errors in the FSSP.

Irregularly shaped particles or ice crystals generally do not pose a severe problem for the OAP. They do not cause counting errors as in the FSSP. It is however difficult to classify irregularly shaped particles with any one number, such as diameter, and their measured size often depends on their orientation in the laser beam.

\section{Optical Effects}

Optical effects determine the operating envelope of the instrument by placing fundamental restrictions on accuracy and precision of the measured size of the water droplet. The FSSP is restricted by such factors as a multivalued Mie response function, over size errors, the laser beam's intensity profile, and the depth of field. The OAP is limited by Fresnel diffraction and depth of field errors.

\section{Mie Response Function}

A plot of the Mie response function for the FSSP is shown in figure 1. It shows the scattered light intensity as a function of droplet diameter. of particular interest is the region where the diameter of the water droplets is on the order of the wavelength of the light. This is called the Mie region of the response function.

In the Mie region the response function is multivalued. Water droplets of different sizes can scatter the same amount of light. This places fundamental limitations on the ability to resolve different diameter water droplets. To help alleviate this problem the FSSP uses a multimodal laser beam. ${ }^{10}$ The effect is to wash out the bumps in the Mie region. There are some questions as to the effectiveness of this technique. ${ }^{2}$ Water droplets of different diameters that produce the same signal may need to be grouped into the same size bin to alleviate the problem.

\section{Over Size Errors}

When Mie theory is used to calculate the scattered intensity from a water droplet, one of the assumptions is the light illuminating the water droplet is a plane wave of uniform intensity. This assumption is valid if the water droplet is much smaller than the diameter of the focused laser beam. For water droplets that are about the same size or larger than the diameter of the laser beam, additional corrections to Mie theory are necessary. ${ }^{11}$

The scattering pattern from large water droplets in a 
gaussian laser beam is quite different from small ones. Figure 2 shows the scattered intensity collected over a solid angle. This plot was made by moving an aperture from a dark field reticle through the cross section of a focused gaussian laser beam. ${ }^{12}$ At each location the aperture scattered light in a manner similar to that of a spherical absorbing particle. The light was collected over a solid angle and the intensity was plotted as a function of position within the beam. The pattern is unusual but can be predicted with Fraunhofer diffraction theory if the gaussian intensity profile of the beam is also modeled in the calculation.

The implication of this is, water droplets that are large and outside of the range of the FSSP could be mistaken as two smaller size water droplets. If a large water droplet traverses the center chord of the laser beam, it will produce a signal with two peaks. This could cause the FSSP to interpret a large water droplet as two smaller ones, thus producing a sizing and a counting error.

\section{Intensity Profile in the FSSP}

In order for the FSSP to measure the diameter of water droplets consistently, the droplets must only be measured when they traverse the central region of constant intensity in the laser beam. As mentioned previously the FSSP defines the central region by determining the transit time of the water droplet in the beam. Water droplets crossing the edge have a shorter transit time and are rejected.

The precision of the FSSP depends on how uniform the intensity is in the probe volume. If there are intensity variations across this region, droplets of the same size can be measured differently depending on their radial trajectory through the beam. ${ }^{13}$ This will cause broadening of the size spectrum.

\section{Depth of Field in the FSSP}

The depth of field in the FSSP is another dimension of the probe volume. The depth of field is determined by comparing the voltages coming from two detectors: a signal detector and an annulus detector. The signal detector is responsible for making a size determination. Light scattered from a water droplet is focused onto this detector. The annulus detector is used to determine if the water droplet is out of the depth of field. If the voltage from this detector is greater than the voltage from the signal detector, then the particle is out of the depth of field and it is rejected.

There are two problems with this method of determining the depth of field. First, there is a variation in the intensity of the incident laser light across the depth of field. This causes the same problem that the radial variations of intensity caused 
in the intensity profile. Water droplets of the same size traversing the laser beam at different axial locations can be sized differently. This causes a broadening of the size spectrum.

The second problem with the depth of field occurs when water droplets are outside of the depth of field. These water droplets can still scatter some light into the signal detector. This results in a sizing error if these droplets are present at the same time a water droplet is in the probe volume being measured. This is a kind of a coincidence error that is unique to the FSSP and has a higher probability of occurring than the coincidence error previously discussed.

Fresnel Diffraction in the OAP

Fresnel diffraction imposes an accuracy limit on the OAP. The OAP uses the shadow boundary to define the edge of a water droplet. Fresnel diffraction blurs this boundary and makes it impossible to determine the edge and thus the exact size of the water droplet. The OAP will always have a sizing uncertainty of at least several micrometers because of this fundamental limitation.

Depth of Field in the OAP

The depth of field in the OAP goes as the square of the diameter of the water droplet. This means that large water droplets remain in focus over a greater distance and can be sized more accurately than small ones. Thus, the probe volume of the OAP is a function of the diameter of the water droplets. When the instrument tabulates the water droplet size distribution there must be a correction for the varying probe volume. Overall the OAP is better at sizing and counting larger water droplets than smaller ones.

\section{Conclusion}

To ensure accurate measurement of water droplet size distributions with the FSSP and the OAP, the operating envelope must not be exceeded. In order to stay within the operating envelope the user must first know which variables can cause errors, identify how these variables can affect the instrument, and finally recognize when any measurement condition has affected the data. If the theoretical and practical limitations of these instruments are not exceeded, they can make accurate, nonintrusive measurements of water droplet size distributions in an icing environment. 


\section{REFERENCES}

1. Hovenac, E.A. (May 1986). Calibration of Droplet Sizing and Liquid Water Content Instruments: Survey and Analysis. NASA CR 175099: $15 \mathrm{pp}$.

2. Pinnick, R.G., D.M. Garvey and L.D. Duncan (September 1981). Calibration of Knollenberg FSSP Light-Scattering counters for Measurement of Cloud Droplets. J Appl Meteor 20: 1049-1057.

3. Cerni, T.A. (1983). Determination of the size and Concentration of Cloud Drops with an FSSP. J Climate Appl Meteor. 22: 1346-1355.

4. Baumgardner, D. (1987). Corrections for the Response Times of Particle Measuring Probes. Proc. 6th Symposium Meteor. Obs. and Instr., New Orleans, La: 148-151.

5. Baumgardner, D., W. Strapp and J.E. Dye (1985) . Corrections for the Forward scattering spectrometer Probe. Part II: Corrections for Coincidence and Dead-Time Losses. J. Atmos. and oceanic Tech. 2: 626-632.

6. Forward Scattering spectrometer Probe, PMS Model FSSP-100, operating and Servicing Manual. Particle Measuring Systems, Inc., Boulder, CO, 1984 .

7. Young, B.W., and W.D. Bachalo (1987). The Direct Comparison of Three In-Flight Droplet sizing Techniques for Pest. Spray Research. International symposium on optical Particle sizing : Theory and Practice, Rouen, France.

8. Bachalo, W.D., M.J. Houser, and J.N. Smith (1986). Evolutionary Behavior of Sprays Produced by Pressure

Atomizers. AIAA 24th Aerospace Science Meeting. Reno, Nevada: paper 86.0296 .

9. Ide, R.F. NASA Lewis Research Center, A personal communication.

10. Knollenberg, R.G. (1976). Three New Instruments for cloud Physics Measurements. Preprints Int. Conf. Cloud Physics, Amer. Meteor. Soc., Boulder, CO: 554-561.

11. Grehan, G., B. Meyhew., and G. Gouesbet (1986). Localized Approx. to the Generalized Lorenz-Mie Theory and its Application to optical Particle sizing. ICALEO 1986 Proceedings. 58: 189196. 
12. Hovenac, E. A. (Nov. 1986). Use of Rotating Reticles for Calibration of Single Particle Counters. ICALEO 1986 Proceedings. 58: $129-134$.

13. Baumgardner, D. (1987). Droplet sizing and counting Limitations of PMS Probes. ILASS 87 1st Annual Conference on Liquid Atomization and Spray Systems. Madison, WI.

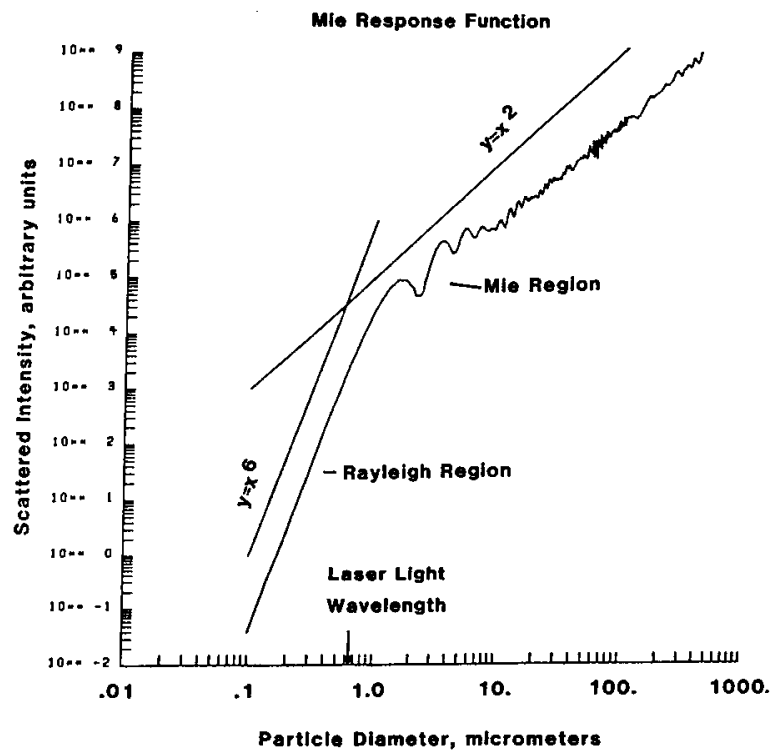

FIGURE 1. - THE MIE RESPONSE FUNCTION FOR THE FSSP.

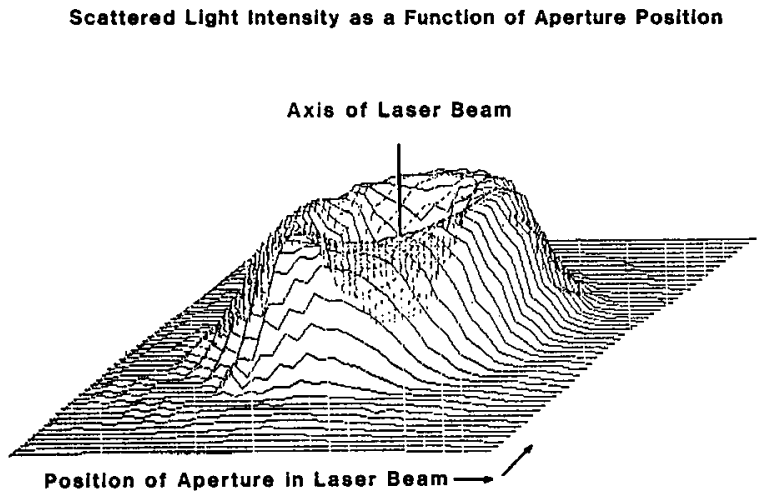

FIGURE 2. - SCATTERED LIGHT COLLECTED OVER A SOLID ANGLE AS A FUNCTION OF THE POSITION OF AN APERTURE WITHIN THE LASER BEAM. 


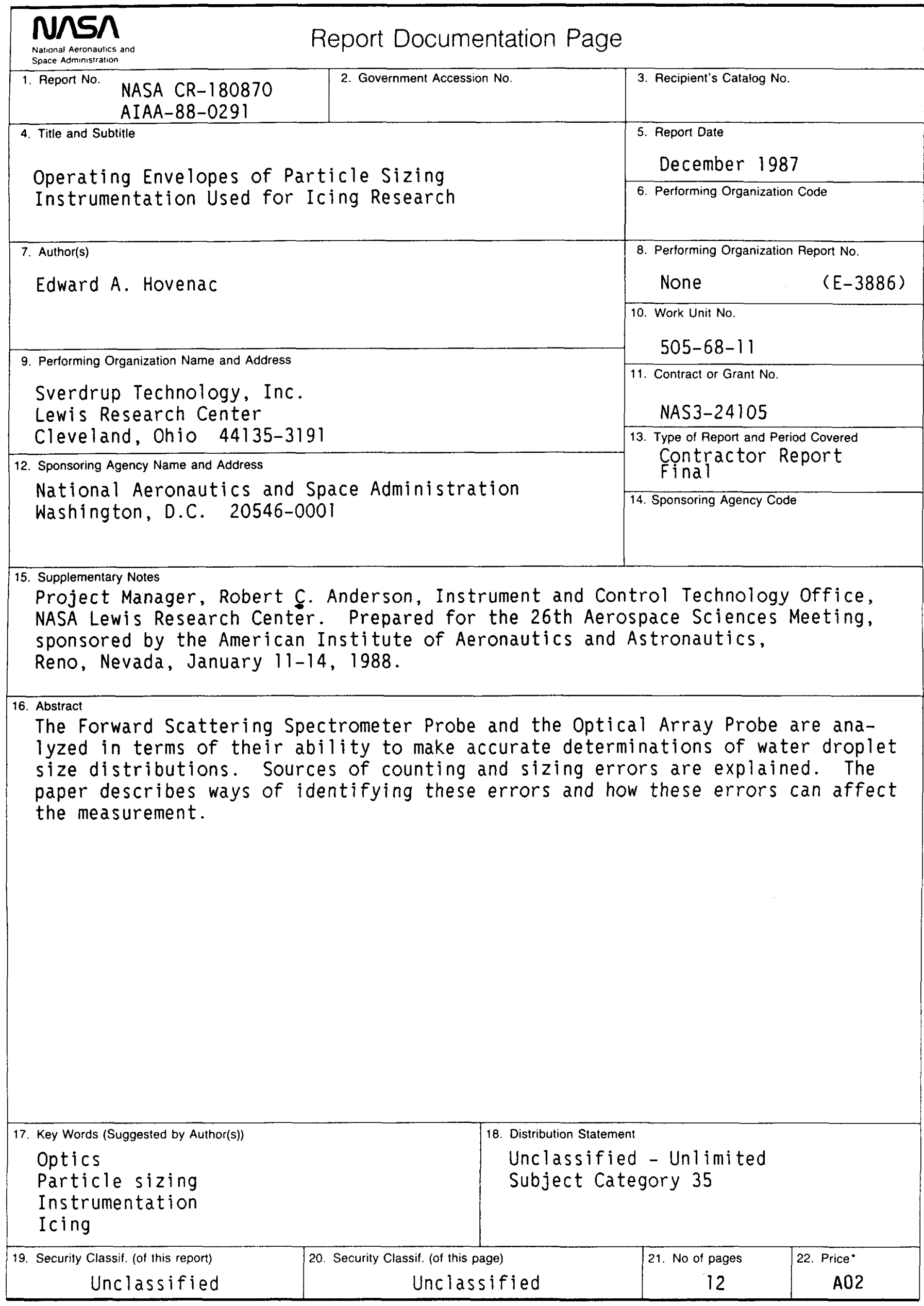

NASA FORM 1626 OCT $86 \quad$ "For sale by the National Technical Information Service, Springfield, Virginia 22161 